\title{
Using Named Entities for Recognizing Family Relationships
}

\author{
E. Oliveira ${ }^{1}$, G. Dias $^{2}$, J. Lima ${ }^{1}$, J.P.C. Pirovani ${ }^{2}$ \\ 1 Laboratório de Computação de Alto Desempenho (LCAD) \\ Universidade Federal do Espírito Santo \\ Av Fernando Ferrari, 514, Goiabeiras - Vitória, ES 29075-910 \\ http://www.lcad.inf.ufes.br/ \\ \{elias, juliana\}@lcad.inf.ufes.br \\ 2 Departamento de Computação \\ Universidade Federal do Espírito Santo \\ Alto Universitário, s/n - Guararema \\ Alegre, ES 29500-000 - Brasil
}

\begin{abstract}
.
Named Entity Recognition problem's objective is to automatically identify and classify entities like persons, places, organizations, and so forth. That is an area in Natural Language Processing and Information Extraction. Named Entity Recognition is important because it is a fundamental step of preprocessing for several applications like relation extraction. However, it is a hard problem to solve as several categories of named entities are written similarly and they appear in similar contexts. To accomplish it, we can use some hybrid approaches. Nevertheless, in this present study, we use linguistic flavor by applying Local Grammar and Cascade of Transducers. Local Grammars are used to represent the rules of a particular linguistic structure. They are often built manually to describe the entities we aim to recognize. In our study, we adapted a Local Grammar to improve the Recognition of Named Entities. The results show an improvement of up to $7 \%$ on the F-measure metric in relation to the previous Local Grammar. Also, we built another Local Grammar to recognize family relationships from the improved Local Grammar. We present a practical application for the extracted relationships using Prolog.
\end{abstract}

CCS Concepts: • Information systems $\rightarrow$ Information extraction.

Keywords: Named-Entity Recognition, Information Extraction, Artificial Intelligence

\section{INTRODUCTION}

A large volume of information these days is available in unstructured free-texts in various sorts of documents, such as a) personals; b) journalistic; c) officials or even d) governmental; and e) in social media. For some automatic applications, in the area of artificial intelligence, it is crucial to extract valuable meta-information from these textual documents. To do this, the Named Entity Recognition (NER) and some other more general Information Extraction (IE) tasks, all of them part of the Natural Language Processing (NLP) area, are important tools. NER aims to identify and classify entities automatically in free written texts, such as names of person, places, organizations, dates, and others, depending on the relevance these domains [Pirovani and Oliveira 2021].

However, NER is not a simple task. The same NE can be classified into different categories depending on the context where it appears. For instance, the NE Washington can refer to the name of a person in one context and one location in another. Another example is the name Rosa Margarida when searched by the Google search engine ${ }^{1}$. It gets confusing to deal with the name of a person and the name of flowers, returning thus both [Lima et al. 2020]. In addition, the same category of NE can be written in different ways in different textual genres. For example, in e-mail texts, it is common to show names of people following words like Olá (Hello in English) and Boa tarde (Good afternoon

\footnotetext{
$\overline{1_{\text {www.google.com }}}$
} 
in English), while in texts of Portarias ( Ordinances in English) and memorandos (Memorandums in English) it is common to show names of people following words like servidor (Oficial in English) and professor (teacher in English). Thus, analyzing texts of different genres can contribute to improving the identification of different rules for the NER.

NER proves useful in several situations, for instance, the search for persons in proceedings or other types of documents, in disambiguation, as shown in the previous examples, in the extraction of relationships [Lima et al. 2020], like a family tree, etc. This task is named as Relationship Extraction $(\mathrm{RE})$.

To evaluate a NER tool, researchers usually adopt the use of benchmark corpus and the previous literature results for comparisons. A corpus may be a set of texts of a given context, or even a variety of domains, such as one or more newspapers, books, among others. HAREM [Mota and Santos 2008; Santos and Cardoso 2007] was an event organized by Linguateca [Linguateca 2018] and was an enormous incentive for NER researchers in the Portuguese language. It adopts the classification of 10 NEs categories, namely, for instance, 1) Abstraction, 2) Event, 3) Thing, 4) Location, 5) Work, 6) Organization, 7) Person, 8) Time, 9) Value, and 10) Other. The annotated corpora used in the HAREM have been used as a golden standard reference for NER systems in Portuguese. These corpora are known as the Golden Collections (GC), and they are split into First HAREM, Mini HAREM, and Second HAREM.

When developing NER systems using the linguistic approach, rules are constructed manually to identify NEs. One strategy for representing the rules of the linguistic approach is the use of Local Grammars (LG), a formalism introduced by Maurice Gross [Gross 1997]. Local grammars are finite-state grammars or finite-state automata that represent sets of expressions in a natural language [Gross 1999]. These grammars are constructed manually and are a manner of grouping or capturing expressions that have common characteristics, whether they are syntactic or semantic.

Pirovani [Pirovani 2019] showed the potential of LGs for use in the NER problem. Both individually when there is no training corpus available, and in conjunction with other machine learning techniques such as Conditional Random Fields (CRF) [Lafferty et al. 2001] to improve its performance. Her work pointed out that any improvements in the LG could also bring improvements to the hybrid approaches.

Therefore, this work, as part of a larger project, has two-folds. Firstly, we sought to explore different textual genres such as emails, ordinances, interviews, and many others in Portuguese to identify rules to be used to adapt an $\mathrm{LG}^{2}$. That is already an improvement in the work carried out by Pirovani [Pirovani 2019; Pirovani et al. 2019]. She built an LG only analyzing the GC of the First HAREM ${ }^{3}$, which contains, in its majority, newspaper texts. In the current work, we will explore a Biblical document genre due to its sophisticated language. Secondly, we proposed a strategy in this work for identifying and annotating the family relationships between pairs of NEs. The family relationships we are interested in are only those of fatherhood and motherhood described in Mathew Chapter 1. In the end, we will have the NEs annotated and a list of parentships cited in worked document.

As a practical application, we present the list of parentships in the Prolog facts format to allow reasoning about them. We also show that it is possible to generate well-structured answers from Prolog facts using LG. The output is an input to another project which aims to provide a robot with an extra logical reasoning capability [Oliveira et al. 2020].

The organization of this article is as follows. In Section 2, we discuss briefly the literature review related to this work. In Section 3, we present the idea of Local Grammar and Cascade of Transducers and we discuss the methodology of our experiments to seek better results. The evaluations carried

\footnotetext{
$2<$ https://inf.ufes.br/ elias/dataSets/ner/recursosTese-julianaPirovani.zip >

${ }^{3}$ Corpus of HAREM available at: www.linguateca.pt/HAREM/
} 
out and the results yielded are presented in Section 4. Our conclusions are in Section 5.

\section{LITERATURE REVIEW}

The NER task can encompass different approaches: linguistic [Pirovani and Oliveira 2015; Rocha et al. 2016], machine learning [Castro et al. 2018; Yang et al. 2017] or hybrid [Pirovani and Oliveira 2018; Pirovani et al. 2019], which can be understood as a combination of the two models.

In their studies, [Pirovani 2019] proposed a model for NER for Portuguese. The authors used a hybrid approach, CRF + LG. The LGs are built using the tool Unitex ${ }^{4}$ [Paumier 2021], a free software package for the construction and application of grammars and cascade. In Unitex, LGs can be represented as one or more graphs. The results of the classification carried out by LG are used as input for a CRF classifier. There is a strong relationship between this work and our study since we propose improvements in the LG proposed by the authors, including using them in cascade.

The approach proposed by [Lima et al. 2018] combines the use of ontologies and logical programming (Prolog) for NER and relation extraction. The approach allows the use of domain ontologies to extract relationships between entities. The model has components for text preprocessing, the generation of a knowledge base; the extraction of new rules; the ontology population. The relationship with our study is in the proposal to create rules in Prolog for relation extraction. The authors worked with binary relations. Among these relations, the kill type relates to two entities of the Person type. Our work presents, in addition to improving the LGs, a proposal for application kinship extraction.

In their work, [Parsaeimehr et al. 2020] proposed a deep learning model for NER and RE. They propose a joint model in order to reduce propagation errors in both tasks. The proposal was developed with four components: embedding layer, Bi-LSTM layer, entity type detection module, and relationship extraction module. For the incorporation layer, the authors used the Glove method. The second component is composed of a recurrent neural network (Bi-LSTM). The third component is responsible for the NER task, and the fourth and last component is responsible for the RE task, which uses a dependency tree and looks for the shortest path between two NEs. The relationship between this study and our work is in using the NER for RE, beyond grammatical structures.

In their studies, [He et al. 2019] proposed a model based on neural networks to extract named entities and kinship relationships. The approach uses a joint neural model with data from online obituaries. In our studies, we also propose a model for extracting kinship relationships. However, our study differs from the proposed model since LG in our work is built using the structure of language and reflects human knowledge, making it possible to explain how to create the rules.

\section{THE METHODOLOGY}

These experiments aim to show our strategies to improve the quality of the identification of NEs and, besides, recognize the relation concept linking a pair of NEs. For the sake of illustration of our experiments, we will focus only on the parenthood relations: fatherhood and motherhood.

\subsection{Extracting Named Entities}

Local Grammar (LG) is a linguistic model, which uses a set of rules built manually [Gross 1997]. Another concept used in our study is the Cascade of Transducers, which is a set of LGs that can be applied in a given order, so that an LG can use the results of the previous one in its rules [Paumier 2021]. The use of cascade has a proposal to solve ambiguity problems generated by LG.

\footnotetext{
$\overline{{ }^{4} \text { https://unitexgramlab.org/en }}$
} 
The LG adapted in this work [Pirovani et al. 2019] was created in Unitex [Paumier 2021] and consists of 10 graphs, one for each of the NEs categories considered by HAREM. The graphs are to capture some simple heuristics for the recognition of NEs.

An example of rule in the graph created for the person category is presented in Figure 1 . This graph recognizes words such as say or said followed by words with the first letter capitalized, as identified by the code <FIRST> in Unitex dictionaries. Among words with the first letter capitalized, prepositions or abbreviations may appear whose recognition has been previously detailed in graphs Preposition.grf and Abbreviation.grf included as subgraphs. An example of occurrence identified by this graph: affirmed <PERSON $>$ José SÓCRATES </PERSON $>$. Note that identified person will appear between the tags $<$ PERSON $>$ and $</$ PERSON $>$ in the concordance file containing the list of occurrences identified.

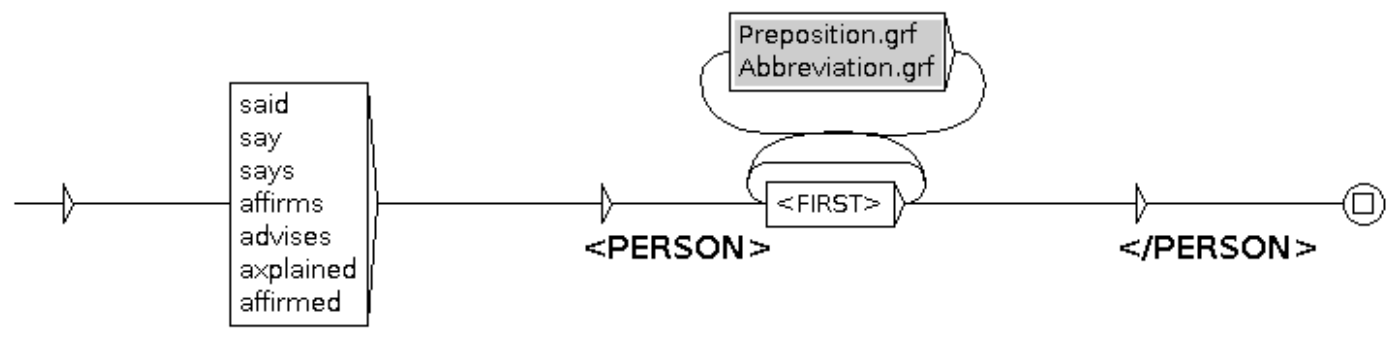

Fig. 1: Example of rule in the graph that recognizes the Person category.

Before starting our experiments, we carried out a linguistic study to identify new rules aiming to improve the LG of Pirovani et al. [Pirovani et al. 2019]. We used the HAREM corpora: PrimeiroHarem, SegundoHarem, and MiniHarem; from a local newspaper A Tribuna, named onwards by aTribuna, several articles; from a governmental institution, documents of Ordinances, and some personal emails. Analyzing these documents, we improved the LG to surpass when the previous LG failed. To find where we should focus on to improve the LG, we looked into each NE's class result, the worst first. We tried to understand when and why the previous approach failed to classify a similar great group of entities and we changed the LG to capture those cases.

One of the first observations when working with the HAREM corpora was that we had to implement some rules including some specific words, such as job's positions and kinships, which precede people's names. Some examples of it would be: the mayor Carlos, the deputy Júlia, among many others.

Another improvement, due to the study of the used datasets, was the inclusion of a rule for the enumeration structure in the written language. The enumeration structure is when NEs of the same category appear in a sequence. The first one is easier to identify from the context where it appears, but its successors do not have a context from which you can classify them. For example, Today, we will have professors João, Ricardo, and Mário at the meeting. João is identified by context, but Ricardo and Mário would not be. Therefore, when an NE is followed by a comma (,) or an $e$ (an and, in English), we assume the next NE, also starting with a capital letter, is an NE from the same class as the first NE.

In addition to these new rules, there also were improvements in the graph referring to the Value entity. Any number followed by a.c. or d.c. is now considered a date. Besides, a number followed by a plural noun is also marked as Value, for instance, 3 apples, 2 men, and so forth. 
The latest improvement was the construction of a Cascade of Transducers using the graphs from the improved LG. We performed some tests in order to find dependencies between the graphs. As a consequence of these tests, it was possible to generate a better order to apply the graphs and take advantage of this method.

Having all NEs annotated within the texts, we can now seek for the relations between entities to annotate as well.

\subsection{Extracting Relations}

The identification of relations between NEs is another goal of this work, although it is still ongoing research. The ultimate goal is to provide search engines [Pirovani et al. 2018] with annotated documents with both NEs and extracted relations for their indexing processes and logical reasoning for a robot [Oliveira et al. 2020].

Our initial focus is on relations that happen only between pairs of NEs. For that, we chose to find fatherhood and motherhood in the Biblical book, the Gospel of Mathew ${ }^{5}$, Chapter 1. There is plenty of that type of relations in this book spanning up to forty-two generations from Abraham to Jesus. The book is challenging because of its formal language, in some versions, and a variety of ways of expressing fatherhood. For instance, the sentence Abraham was the father of Isaac, in verse 2, and Jacob the father of Joseph, the husband of Mary, and Mary was the mother of Jesus who is called the Messiah, in verse 16, all these examples from the same a translation version in English.

Figure 2 shows the pipeline of the process. First, the NER is performed by applying the Cascade of Transducers mentioned in the previous section, recognizing all the NEs of interest. Latter, from the chunk of sentence between a pair of NEs, we extracted the relation of interest, in this work only the parenthood. The relationships were extracted from a LG built for this which was added to the Cascade. An example of a rule in this LG is presented in Figure 3. Note that this LG uses the results of NER (<PERSON> captures entities of the Person category previously recognized by the Cascade). A syntax transformation is needed to uniforms the relational predicate along with the knowledge database. Note thus that the quality of NER plays a crucial role in the whole proposed approach.

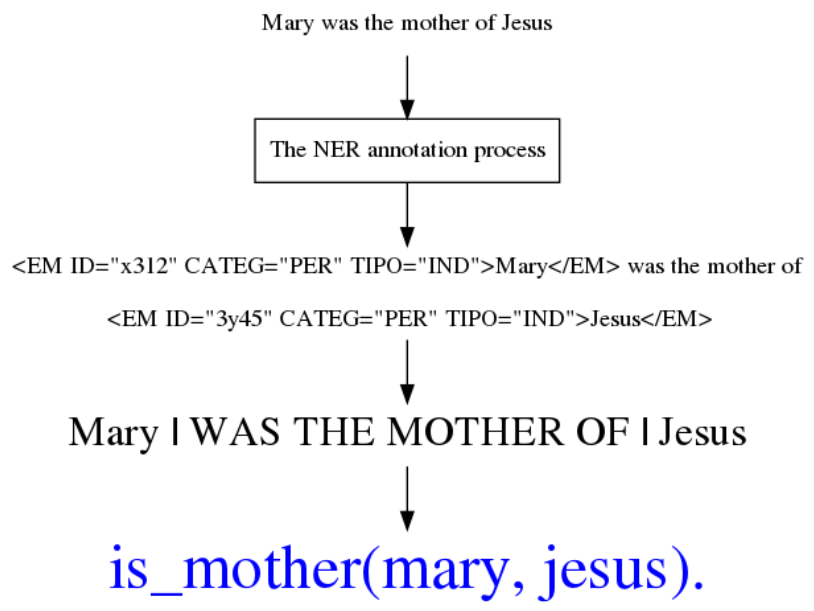

Fig. 2: The pipeline of the process

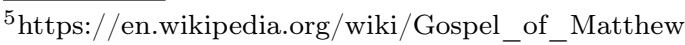




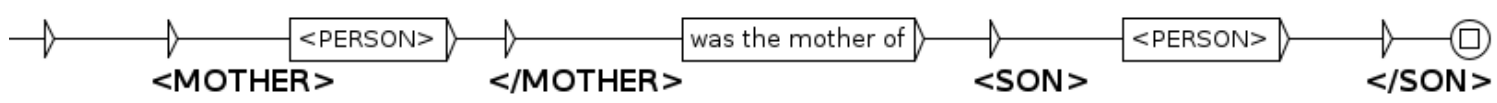

Fig. 3: Example of rule in the LG that extracts relationships

\begin{tabular}{lllll}
\hline \multicolumn{1}{c}{ Approach } & \multicolumn{1}{c}{ Corpus } & Precision & Recall & F-Measure \\
\hline \multirow{3}{*}{ 1) LG of Pirovani et al. [Pirovani et al. 2019] } & PrimeiroHarem & 0.70 & 0.43 & 0.53 \\
& SegundoHarem & 0.70 & 0.39 & 0.50 \\
& MiniHarem & 0.67 & 0.41 & 0.51 \\
\hline \multirow{3}{*}{ 2) Adapted LG } & PrimeiroHarem & 0.71 & 0.41 & 0.52 \\
& SegundoHarem & 0.71 & 0.45 & 0.55 \\
& MiniHarem & 0.68 & 0.42 & 0.52 \\
\hline \multirow{3}{*}{ 3) Cascade of Transducers } & PrimeiroHarem & 0.72 & 0.49 & 0.59 \\
& SegundoHarem & 0.71 & 0.44 & 0.54 \\
& MiniHarem & 0.72 & 0.48 & 0.58 \\
\hline \hline
\end{tabular}

Table I: The results of each approach

\section{RESULTS}

The results of the application of each approach in the corpora PrimeiroHarem, SegundoHarem, and MiniHarem are shown in Table I. In the last columns, we placed the metrics used in these evaluations: Precision, Recall, and F-measure, the average of the previous two metrics. In other corpora used in our linguistic studies it was not possible to obtain the results of the metrics adopted, due to the lack of human annotations for comparison, or the lack of some of the analyzed entities, those discussed in Section 1. Even without the results of the metrics, we do not fail to observe the behavior of the proposal LG and Cascade in such corpus.

The results of the application of the LG built in Pirovani et al. [Pirovani et al. 2019] are shown in Table I (1). With all the changes presented in the Section 3.1, we yielded the following result shown in Table I (2). Applying the Cascade of Transducers, we obtained the results shown in Table I (3). Note that in the previous results, a direct application was used initially, without observing which order would be the best for the application of the graphs. These results with the Cascade show a gain of 7 percentage points in F-measure for the corpus Mini-HAREM in comparison to the original LG. We believe that the Cascade approach performed better because it: a) uses previous results; b) adds more contextual information in some situations; and c) prevents from some rules to generate ambiguities.

Table II shows part of the basic relational genealogic facts we extracted from the Chapter of Mathew 1, after the execution of the pipeline shown in Figure 2.Analyzing the results in the cited chapter, the Cascade was able to extract all the mentioned relationships between father and mother and their respective children. In the color blue, we depicted the motherhood we could find in the text. Just a reminder that it was unusual at that time to register facts related to any woman. The first fatherhood is in boldface, on top left of the table: is father(abraham, isaac). According to the Bible, Abraham is called the father of all the Jews people. The last fatherhood, before Jesus, is given at the bottom right of the table: is_father(joseph, jesus). With all this information we can now infer who should be the grandfather of Jesus, for example.

In the color red, we highlight a challenging situation that may occur in any other domain but is frequent in the Bible. Someone is said to be the father of another person figuratively. That is the case when David is called to be the son of Abraham. The fact which is represented by the Prolog tuple as is father(abraham, david). Almost at the end of the table, another expression, in red, saying that David is the father of Jesus Christ. There are two important things to point out in this example: a) the first is when someone else is said to be the father of Jesus, but b) Jesus is given 




Table II: Part of the found list of parenthood in the book of Mathew 1

another name, for instance, Jesus Christ. Would it be another person or the same Jesus mentioned previously? That is one of the disambiguation challenges we also need to tackle to extract the correct fact and thus be able to reason correctly over them.

From the Prolog facts database, it is also possible to generate answers using LG. Figure 4 shows an LG that generates, for example, the answer joseph is the father of Jesus for the Prolog's predicate is father(joseph,jesus). This LG uses variables (father and son in red) and appends the necessary sentence-text for the answer in the desired format to the output. In this case, only the is father predicate is recognized, but similar LGs can generate responses to other Prolog facts. It is also possible to build a generic graph that generates answers to any predicates provided that their structure is similar and known a priori.

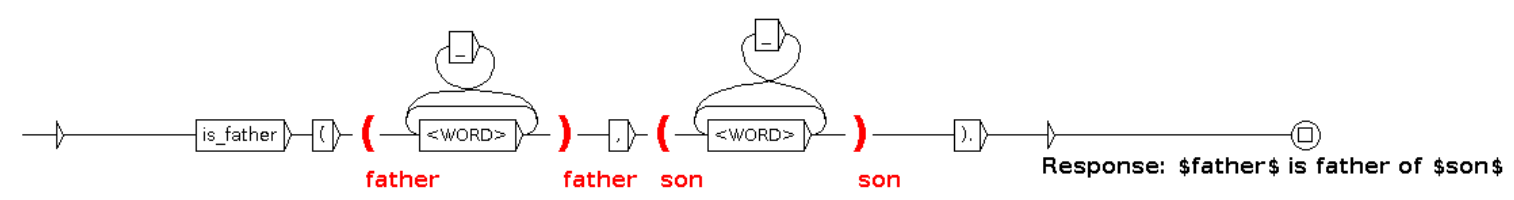

Fig. 4: LG that generates answers

\section{CONCLUSIONS}

This work aimed to present a methodology stemming from the linguistic procedures, which are very good for situations where we do not have a reasonable amount of training dataset for the supervised algorithms [Fonseca et al. 2018]. On the other hand, with this methodology, we greatly facilitate the rapid generation of an annotated database for then create a training dataset for the supervised algorithms [Lima et al. 2020], when that is the goal.

We describe in this work some efforts to improve the performance of an LG [Pirovani et al. 2019]. The grammatical rules, now part of the LG, were built with the features of software Unitex [Paumier 2021]. These improvements also included the creation of a Cascade of Transducers. The Cascade approach performed better. We observed an improvement of up to $5 \%$ among the best versions of LG and Cascade, over the result presented in [Pirovani et al. 2019].

We also presented in this paper the use of LG to extract the parentship between pairs of NEs. In order to illustrate the execution of the pipeline proposed in this work, we focused only on the 
Biblical book of Mathew, Chapter 1. The same applies to any other document. We generate a list of parentship in the Prolog facts format to allow other applications [Oliveira et al. 2020] of reasoning over these logical facts.

This work is ongoing long-term research on understanding the use of LG as a resort when there are no training datasets available, but also to improve supervised approaches. Another goal, as future work, is to use the annotated NEs both to create more sophisticated logical-knowledge datasets, and to generate exam questions, as proposed in [Pirovani et al. 2017].

\section{ACKNOWLEDGMENT}

The authors thank the Fundo de Apoio à Pesquisa da Universidade Federal do Espírito Santo (FAP/UFES) for the scholarship awarded to Gabriel Magalhães Dias. The first author was partially supported by Vitoria Virtual ${ }^{6}$ a local Brazilian startup.

\section{REFERENCES}

Castro, P. V. Q., Silva, N. F. F., And Soares, A. S. Portuguese Named Entity Recognition Using LSTM-CRF. In Villavicencio A. et al. (eds) Computational Processing of the Portuguese Language. PROPOR 2018. Lecture Notes in Computer Science, vol 11122. Springer, Cham, Canela, RS, pp. 83-92, 2018.

Fonseca, E., Medeiros, I., Kamikawachi, D., And Bokan, A. Automatically Grading Brazilian Student Essays. In International Conference on Computational Processing of the Portuguese Language. Springer, pp. 170-179, 2018.

Gross, M. The Construction of Local Grammars. In ROCHE, E.; SCHABES, Y. (eds.). Finite-State Language Processing, Language, Speech, and Communication, Cambridge, Mass., 1997.

Gross, M. A Bootstrap Method for Constructing Local Grammars. In Proceedings of the Symposium on Contemporary Mathematics. University of Belgrad, pp. 229-250, 1999.

He, K., Wu, J., Ma, X., Zhang, C., Huang, M., Li, C., and Yao, L. Extracting Kinship from Obituary to Enhance Electronic Health Records for Genetic Research. In Proceedings of the Fourth Social Media Mining for Health Applications (\# SMM4H) Workshop \& Shared Task. pp. 1-10, 2019.

Lafferty, J., McCallum, A., and Pereira, F. Conditional Random Fields: Probabilistic Models for Segmenting and Labeling Sequence Data. In Proceedings of the Eighteenth International Conference on Machine Learning, ICML 2001. Vol. 1. pp. 282-289, 2001.

Lima, J., Colombo, C., Izo, F., , Oliveira, E., and Badue, C. Finding Entities and Related Facts in Newspaper. In 20th International Conference on Intelligent Systems Design and Applications - (ISDA). Springer, Springer International Publishing, On the WWW, pp. 102-113, 2020.

Lima, J., Colombo, C., Izo, F., Pirovani, J. C. P., And Oliveira, E. Using CRF+LG for Automated Classification of Named Entities in Newspaper Texts. In Computing Conference (CLEI), 2020 Latin American. IEEE, Loja, Ecuador, 2020.

Lima, R., Espinasse, B., And Freitas, F. OntoILPER: an Ontology and Inductive Logic Programming-Based System to Extract Entities and Relations from Text. Knowledge and Information Systems 56 (1): 223-255, 2018.

LinguatecA., 2018. Acesso em: 17/06/2021.

Mota, C. and Santos, D. Desafios na Avaliação Conjunta do Reconhecimento de Entidades Mencionadas: O Segundo HAREM. Linguateca, 2008.

Oliveira, E., Spalenza, M., And Pirovani, J. rAVA: A Robot for Virtual Support of Learning. In 20 th International Conference on Intelligent Systems Design and Applications - (ISDA). Springer, Springer International Publishing, On the WWW, pp. 102-113, 2020.

Parsaeimehr, E., Fartash, M., and Torkestani, J. A. An Enhanced Deep Neural Network-Based Architecture for Joint Extraction of Entity Mentions and Relations. International Journal of Fuzzy Logic and Intelligent Systems 20 (1): 69-76, 2020.

Paumier, S. Unitex 3.2 User Manual, 2021. Acesso em: 24/06/2021.

Pirovani, J., Alves, J., Spalenza, M., Silva, W., Silveira Colombo, C., and Oliveira, E. Adapting Ner $(\mathrm{CRF}+\mathrm{LG})$ for Many Textual Genres. In Proceedings of the Iberian Languages Evaluation Forum (IberLEF 2019). CEUR Workshop Proceedings, vol. 2421. CEUR-WS.org, Bilbao, Spain, pp. 421-433, 2019.

Pirovani, J., Nogueira, M., And Oliveira, E. Indexing Names of Persons in a Newspaper Large Dataset. In $13^{\text {th }}$ International Conference on the Computational Processing of Portuguese (PROPOR). Vol. 11122. Springer, Canela, RS, 2018.

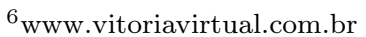


Pirovani, J. and Oliveira, E. Portuguese Named Entity Recognition Using Conditional Random Fields and Local Grammars. In LREC. European Language Resources Association (ELRA), Miyazaki, Japan, pp. 4453-4456, 2018.

Pirovani, J. And Oliveira, E. Studying the Adaptation of Portuguese NER for Different Textual Genres. The Journal of Supercomputing, 2021.

Pirovani, J., Spalenza, M., And Oliveira, E. Geração Automática de Questões a Partir do Reconhecimento de Entidades Nomeadas em Textos Didáticos. In XXVIII Simpósio Brasileiro de Informática na Educação (SBIE). SBC, Ceará, CE, pp. 1147-1156, 2017.

Pirovani, J. P. C. CRF+LG: Uma Abordagem Híbrida para o Reconhecimento de Entidades Nomeadas em Português. Ph.D. thesis, Programa de Pós-Graduação em Informática, Universidade Federal do Espírito Santo, Vitória, ES, 2019.

Pirovani, J. P. C. and Oliveira, E. Extração de Nomes de Pessoas em Textos em Português: uma Abordagem Usando Gramáticas Locais. In Computer on the Beach 2015. SBC, Florianópolis, SC, 2015.

Rocha, C., Jorge, A., Sionara, R., Brito, P., Pimenta, C., and Rezende, S. PAMPO: Using Pattern Matching and Pos-tagging for Effective Named Entities Recognition in Portuguese, 2016.

Santos, D. and Cardoso, N. Reconhecimento de Entidades Mencionadas em Português: Documentação e Actas do HAREM, a Primeira Avaliação Conjunta na Área. Linguateca, 2007.

YAng, J., Zhang, Y., And Dong, F. Neural Reranking for Named Entity Recognition. arXiv preprint arXiv:1707.05127, 2017. 